EPHOU-93-002

November, 1993

\title{
A Mean Field Analysis of One Dimensional Quantum Liquid with Long Range Interaction
}

\author{
Kenzo Ishikawa and Nobuki Maeda \\ Department of Physics, Hokkaido University, \\ Sapporo, 060 Japan
}

\begin{abstract}
Bi-local mean field theory is applied to one dimensional quantum liquid with long range $1 / r^{2}$ interaction, which has exact ground state wave function. We obtain a mean field solution and an effective action which expresses a long range dynamics. Based on them the ground state energy and correlation functions are computed. The ground state energy agrees fairly well with the exact value and exponents have weaker coupling constant dependence than that of partly known exact value.
\end{abstract}


Recently there are much interests in one dimensional quantum liquid with $1 / r^{2}$ potential. Ground state wave function and thermodynamic quantities were found by Sutherland [1] and Calogero [2]. Ground state wave function is of Jastrow type and becomes simple form in certain values of coupling constant. It is possible to compute exponents of correlation functions for these values of coupling constant. For other general values it has been impossible to compute them. Kawakami and Yang[3] computed recently the exponents based on conformal field theory. They show that the system belongs to Luttinger liquid [4] [6].

In the present paper we apply a bi-local mean field theory. Mean field theory has been successfully applied to many places. It is not clear if a mean field theory can be applied to strongly correlated systems [7] [8]. We apply such a mean field theory that includes correlations and compare results with partly known exact values. We see that a fair agreement is obtained in the ground state energy but an agreement is not good for the exponents of correlation functions if the linearized action is used.

Lagrangian which discribes interacting fermion system is

$$
\mathcal{L}=\psi^{\dagger}\left(i \frac{\partial}{\partial t}-e A_{0}\right) \psi-\psi^{\dagger} \frac{(\vec{p}+e \vec{A})^{2}}{2 m} \psi-\int d \vec{y} \psi^{\dagger}(x) \psi^{\dagger}(y) \frac{V(\vec{x}-\vec{y})}{2} \psi(y) \psi(x),
$$

where $A_{0}$ and $\vec{A}$ are external electromagnetic potentials and $V(x-y)$ is the two body potential energy between electrons. We concentrate on the repulsive long range interaction, $V(\vec{x}-\vec{y})=g /|\vec{x}-\vec{y}|^{2}$. Green's functions are computed from the partition function as

$$
\mathcal{Z}=\int \mathcal{D} \psi^{\dagger} \mathcal{D} \psi e^{-\int_{0}^{\beta} d \tau \int d x \mathcal{L}_{E}}
$$

where $\beta=1 / k T$ and $\tau=i t$ in the path integral quantization method. The fermionic fields $\psi(x)$ and $\psi^{\dagger}(x)$ are anti-commuting c-numbers. We write the interaction term as

$$
\begin{gathered}
-\frac{1}{2} \psi^{\dagger}(x) \psi(y) \frac{V(x-y)}{2} \psi^{\dagger}(y) \psi(x)(2-p(x-y)) \\
+\frac{1}{2} \psi^{\dagger}(x) \psi(x) \frac{V(x-y)}{2} \psi^{\dagger}(y) \psi(y) p(x-y),
\end{gathered}
$$

where $p(x-y)$ is a c-number function and a suitable form is chosen depending upon the interaction and dimension of space. In the present paper we use $p(x-y)=1$.

Let us describe the system with one spatial dimension here, but its extension is straightforward. We rewrite the partition function 9] using bi-local auxiliary field $U\left(x_{1}, y_{1} ; x_{0}\right)$ that is local in time coordinate and bi-local in space coordinate.

$$
\mathcal{Z}=\frac{1}{N} \int \mathcal{D} U \mathcal{D} \psi^{\dagger} \mathcal{D} \psi e^{-\int d x_{0} d x_{1}\left[\mathcal{L}_{0}+\int d y_{1} \mathcal{L}_{e f f}\right]}
$$




$$
\begin{aligned}
\mathcal{L}_{0}=\psi^{\dagger}(x)\left(\frac{\partial}{\partial x_{0}}+e A_{0}\right) \psi(x)+\psi^{\dagger}(x) \frac{\left(p_{1}+e A_{1}\right)^{2}}{2 m} \psi(x), & \\
\mathcal{L}_{e f f}= & {\left[\left\{U\left(x_{1}, y_{1} ; x_{0}\right) U\left(y_{1}, x_{1} ; x_{0}\right)-U\left(x_{1}, y_{1} ; x_{0}\right) \psi^{\dagger}(y) \psi(x)\right.\right.} \\
& \left.\quad-U\left(y_{1}, x_{1} ; x_{0}\right) \psi^{\dagger}(x) \psi(y)\right\}-\left\{U\left(x_{1}, x_{1} ; x_{0}\right) U\left(y_{1}, y_{1} ; x_{0}\right)\right. \\
& \left.\left.-U\left(x_{1}, x_{1} ; x_{0}\right) \psi^{\dagger}(y) \psi(y)-U\left(y_{1}, y_{1} ; x_{0}\right) \psi^{\dagger}(x) \psi(x)\right\}\right] \frac{1}{4} V(x-y), \\
N= & \int \mathcal{D} U e^{-\int d x_{0} d x_{1} d y_{1} \frac{V\left(x_{1}-y_{1}\right)}{4}\left\{U\left(x_{1}, y_{1} ; x_{0}\right) U\left(y_{1}, x_{1} ; x_{0}\right)-U\left(x_{1}, x_{1} ; x_{0}\right) U\left(y_{1}, y_{1} ; x_{0}\right)\right\}} .
\end{aligned}
$$

The partition function goes back to the Eq.(2) by integrating the bi-local field $U\left(x_{1}, y_{1} ; x_{0}\right)$ in Eq.(4). We write $\mathcal{Z}$, by integrating the fermionic field first, as

$$
\begin{gathered}
\mathcal{Z}=\frac{1}{N} \int \mathcal{D} U e^{-S_{e f f}(U)} \\
e^{-S_{e f f}(U)}=\int \mathcal{D} \psi^{\dagger} \mathcal{D} \psi e^{-\int d x_{0} d x_{1}\left[\mathcal{L}_{0}+\int d y_{1} \mathcal{L}_{e f f}\right]} .
\end{gathered}
$$

(A) Stationary phase approximation - The integration of the bi-local field is defined around a minimum of the classical action $S_{\text {eff }}(U)$, i.e., a mean field $U_{0}\left(x_{1}, y_{1}\right)$ which satisfies a self-consistency condition,

$$
\begin{gathered}
\left.\frac{\partial S_{e f f}(U)}{\partial U}\right|_{U=U_{0}}=0, \\
\left\langle\psi^{\dagger}(x) \psi(y)\right\rangle_{\substack{x_{0}=y_{0} \\
U=U_{0}}}=U_{0}\left(x_{1}, y_{1}\right),
\end{gathered}
$$

in stationary phase approximation. If $U_{0}\left(x_{1}, y_{1}\right)$ is a function of $x_{1}-y_{1}$, the system is invariant under translation and momentum is a good quantum number. The ground state in a mean field approximation is defined as a state vector in which one particle states up to Fermi momentum are filled. Hence the functions

$$
\begin{gathered}
A_{0}=\mu, A_{1}=0, \\
U_{0}\left(x_{1}, y_{1}\right)=\frac{\sin \pi r \rho}{\pi r},
\end{gathered}
$$

where $r=x_{1}-y_{1}$, satisfy the self-consistency condition. $\rho$ is the density and is connected with the Fermi momentum by $p_{F}=\pi \rho$ and $\mu$ is the chemical potential. Using the above $U_{0}\left(x_{1}, y_{1}\right)$ of Eq.(8), $\mathcal{Z}$ is written as $\mathcal{Z}=\mathcal{Z}_{0} \tilde{\mathcal{Z}}$

$$
\begin{gathered}
\mathcal{Z}_{0}=e^{-S_{\text {eff }}\left(U_{0}\right)}, \\
\tilde{\mathcal{Z}}=\frac{1}{N \mathcal{Z}_{0}} \int \mathcal{D} U \mathcal{D} \psi^{\dagger} \mathcal{D} \psi e^{-S\left(U_{0}+U, \psi^{\dagger}, \psi\right)},
\end{gathered}
$$




$$
S\left(U, \psi^{\dagger}, \psi\right)=\int d x_{0} d x_{1}\left(\mathcal{L}_{0}+\int d y_{1} \mathcal{L}_{e f f}\right)
$$

The mean field part, $\mathcal{Z}_{0}$, corresponds to the mean field Hamiltonian,

$$
\begin{gathered}
H_{m}=\int d x_{1}\left[\psi^{\dagger}(x) \frac{p_{1}^{2}}{2 m} \psi(x)+\psi^{\dagger}(x) F\left(p_{1}\right) \psi(x)\right]+E_{\text {background }}, \\
F\left(p_{1}\right)=-2 \int d r \frac{V(r)}{4}\left\{U_{0}(r) e^{i p_{1} r}-U_{0}(0)\right\}, \\
E_{\text {background }}=\int d x_{1} d y_{1} \frac{V(x-y)}{4}\left\{\left|U_{0}\left(x_{1}-y_{1}\right)\right|^{2}-U_{0}(x, x) U_{0}(y, y)\right\} .
\end{gathered}
$$

The second term of the kinetic energy and the background energy are generated from the mean field part and have the following forms,

$$
\begin{gathered}
F(p)=\left\{\begin{array}{cc}
g\left(p^{2}+p_{F}^{2}\right) / 4, & |p|<p_{F}, \\
g p_{F}|p| / 2, & |p|>p_{F},
\end{array}\right. \\
E_{\text {background }}=-\int d x_{1} \frac{g}{6} \frac{p_{F}^{3}}{\pi} .
\end{gathered}
$$

In mean field approximation, in which we take only $\mathcal{Z}_{0}$, the energy density, the fermion propagator and the density correlation function become as

$$
\begin{gathered}
\mathcal{E}_{\text {mean field }}=\int_{-p_{F}}^{p_{F}} \frac{d p}{2 \pi}\left\{\frac{p^{2}}{2 m}+F(p)\right\}-\frac{g}{6} \frac{p_{F}^{3}}{\pi}=\frac{1}{6 \pi m} p_{F}^{3}+\frac{g}{6} \frac{p_{F}^{3}}{\pi}, \\
\left\langle\psi^{\dagger}\left(x_{1}, x_{0}\right) \psi\left(y_{1}, x_{0}\right)\right\rangle=\frac{\sin p_{F} r}{\pi r} \\
\left\langle\rho\left(x_{1}, x_{0}\right) \rho\left(y_{1}, x_{0}\right)\right\rangle=\frac{1}{2 \pi^{2}} \frac{1}{r^{2}}-\frac{1}{2 \pi^{2}} \frac{\cos 2 p_{F} r}{r^{2}} .
\end{gathered}
$$

Obviously the self-consistency condition Eq.(7) is satisfied.

$\tilde{\mathcal{Z}}$ in Eq.(9) is due to the fluctuations around the mean field, and gives a correction to the above energy density and propagator. We compute their effects in the following using local field expansions of the bi-local field. The bi-local field is expanded by a complete set of relative coordinates as

$$
\begin{gathered}
U\left(x_{1}, y_{1} ; x_{0}\right)=U_{0}\left(x_{1}-y_{1}\right)\left[1+\sum_{n=0}^{\infty} q_{n}\left(x_{1}-y_{1}\right) a_{n}\left(\frac{x_{1}+y_{1}}{2}, x_{0}\right)\right] \\
\int d r \frac{V(r)}{4}\left[\left|U_{0}(r)\right|^{2} q_{n}(r) q_{m}(-r)-\left|U_{0}(0)\right|^{2} q_{n}(0) q_{m}(0)\right]=\delta_{n, m}, \\
q_{0}=i C_{0}, q_{1}=i C_{1} r ; C_{0}, C_{1} \text { real, } \\
q_{2 n}=2 \mathrm{n} \text {-th real polynomials }
\end{gathered}
$$




$$
q_{2 n+1}=i \times(2 \mathrm{n}+1 \text {-th real polynomials }) .
$$

For a computational convenience, we modify the long range $g / r^{2}$ potential to a short range potential, $g e^{-\epsilon r^{2}} / r^{2}$ and let $\epsilon \rightarrow 0$ at the end. We see that some of the local fields decouple. This complete set is constructed by Schmidt orthogonalization method starting from a set of functions $\left\{1, r, r^{2}, \cdots\right\}$. With the above normalization of $q_{n}(r)$, the mass terms become diagonal forms of the local fields $a_{n}(x)$, and the effective action is written as,

$$
\begin{gathered}
S\left(U, \psi, \psi^{\dagger}\right)=\int d x_{1}\left[\left(\frac{1}{q_{0}}+a_{0}(x)\right)^{2}+a_{1}(x)^{2}+a_{2}(x)^{2}+\cdots\right. \\
+\left(\frac{1}{q_{0}}+a_{0}(x)\right) \psi^{\dagger}(x) F_{0}(p) \psi(x)+\sum_{l=1} a_{l}(x) \psi^{\dagger}(x) F_{l}(p) \psi(x) \\
+\sum_{n=0} q_{n}(0) a_{n}(x) C_{0}(p) \sum_{m=0} q_{m}(0) a_{m}(x) \\
+2\left\{\frac{C_{0}(p)}{U_{0}} \sum_{l=0} q_{l}(0) a_{l}(x)\right\} \psi^{\dagger}(x) \psi(x)-\psi^{\dagger}(x) \frac{\partial}{\partial x_{0}} \psi(x)+\psi^{\dagger}(x) \frac{p^{2}}{2 m} \psi(x)+\mu \psi^{\dagger}(x) \psi(x)
\end{gathered}
$$

The coupling strengths of local fields are given by

$$
\begin{gathered}
F_{l}(p)=-2 \int d r\left\{U_{0}(r) e^{i p r} q_{l}(r)-U_{0}(0) q_{l}(0)\right\} \frac{V(r)}{4} \\
C_{0}(p)=\int d r\left|U_{0}(0)\right|^{2} \frac{V(r)}{4}\left(e^{i p r}-1\right) .
\end{gathered}
$$

The low energy and long distance physics are determined by $F_{l}\left(p_{F}\right)$. They behave for $l \geq 2$, as

$$
F_{l}\left(p_{F}\right)=\text { const } \epsilon^{\frac{1}{4}} \quad(l \geq 2),
$$

when the modified form of the potential is used. Obviously $F_{l}\left(p_{F}\right)(l \geq 2)$ vanish in the small $\epsilon$ limit, and $a_{l}(x)(l \geq 2)$ decouple.

Hereafter, we take only $a_{0}(x)$ and $a_{1}(x)$ into account and study the system with them. The action then is given by

$$
\begin{gathered}
S\left(a_{0}, a_{1}, \psi, \psi^{\dagger}\right)=\int d x\left[\left(\frac{1}{q_{0}}+a_{0}(x)\right)^{2}+a_{1}(x)^{2}\right. \\
+q_{0}^{2} a_{0}(x) C_{0}(p) a_{0}(x)+\left(\frac{1}{q_{0}}+a_{0}(x)\right) \psi^{\dagger}(x) F_{0}(p) \psi(x)+a_{1}(x) \psi^{\dagger}(x) F_{1}(p) \psi(x) \\
\left.+2\left\{\frac{C_{0}(p)}{U_{0}} q_{0} a_{0}(x)\right\} \psi^{\dagger}(x) \psi(x)-\psi^{\dagger}(x) \frac{\partial}{\partial x_{0}} \psi(x)+\psi^{\dagger}(x) \frac{p^{2}}{2 m} \psi(x)+\mu \psi^{\dagger}(x) \psi(x)\right]
\end{gathered}
$$


We consider fluctuations of the fermion field of momentum near the Fermi momenta $\pm p_{F}$, and use the following linearization approximation :

$$
\begin{gathered}
F_{0}(p)=F_{0}\left( \pm p_{F}\right)+\left(p \mp p_{F}\right) F_{0}^{\prime}\left( \pm p_{F}\right), \\
a_{0}(x) F_{0}(p)=a_{0}(x) F_{0}\left( \pm p_{F}\right) \\
\frac{p^{2}}{2 m}=\frac{p_{F}^{2}}{2 m}+\left(p \pm p_{F}\right) \frac{ \pm p_{F}}{m} .
\end{gathered}
$$

The chemical potential makes single-body energy vanishes at the Fermi momenta $\pm p_{F}$.

It is convenient to introduce chiral fields $\psi_{L}$ and $\psi_{R}$ that have a momentum near $p_{F}$ or a momentum near $-p_{F}$. Two chiral fields are further combined into a two component Dirac field and the action is written as

$$
\begin{gathered}
S=C \int d \bar{x}\left[\frac{1}{v_{0}^{2}}\left\{\frac{1}{1+2 C_{0}(p) q_{0} /\left(U_{0} F_{0}\left(p_{F}\right)\right)} \bar{a}_{0}(x)\right\}^{2}+\frac{1}{v_{1}^{2}} \bar{a}_{1}^{2}(x)+q_{0}^{2} a_{0}(x) C_{0}(p) a_{0}(x)\right. \\
\left.+\bar{\psi} \gamma_{0}\left(p_{0}+\overline{a_{0}}\right) \psi+\bar{\psi} \gamma_{1}\left(\overline{p_{1}}+\overline{a_{1}}\right) \psi\right],
\end{gathered}
$$

where

$$
\begin{gathered}
\bar{a}_{0}(x)=\left\{F_{0}\left(p_{F}\right)+2 \frac{C_{0}(p) q_{0}}{U_{0}}\right\} a_{0}(x) \\
\bar{a}_{1}(x)=F_{1}\left(p_{F}\right) a_{1}(x), \\
\bar{p}_{1}=p_{1} C, \gamma_{0}=i\left(\begin{array}{cc}
0 & 1 \\
1 & 0
\end{array}\right), \gamma_{1}=\left(\begin{array}{cc}
0 & 1 \\
-1 & 0
\end{array}\right), \psi=\left(\begin{array}{c}
\psi_{L} \\
\psi_{R}
\end{array}\right), \bar{\psi}=\psi^{\dagger} i \gamma_{0}, \\
v_{0}=F_{0}\left(p_{F}\right), v_{1}=F_{1}\left(p_{F}\right), d \bar{x}=d x_{0} d x_{1} C^{-1}, C=\frac{F_{0}^{\prime}\left(p_{F}\right)}{q_{0}}+\frac{p_{F}}{m} .
\end{gathered}
$$

The action is reduced, further to

$$
\begin{gathered}
S_{e f f}=\int d \bar{x}\left[\frac{C}{v_{0}^{2}}\left(\bar{a}_{0}(x)^{2}+r^{2} \bar{a}_{1}(x)^{2}\right)+\frac{1}{4 \pi} \bar{f}_{\mu \nu} \frac{1}{\bar{\partial}^{2}} \bar{f}_{\mu \nu}+\text { higher derivatives }\right] \\
=\int d \bar{x}\left[C \frac{1}{v_{0}^{2}}\left\{\left(\partial_{0} \lambda\right)^{2}+r^{2}\left(\bar{\partial}_{1} \lambda\right)^{2}+r^{2}\left(\partial_{0} \phi\right)^{2}+\left(\bar{\partial}_{1} \phi\right)^{2}+2\left(1-r^{2}\right) \partial_{0} \lambda \bar{\partial}_{1} \phi\right\}+\frac{1}{2 \pi}\left(\bar{\partial}_{\mu} \phi\right)^{2}\right. \\
\left.+\frac{1}{2 \pi}\left(\bar{\partial}_{\mu} \phi\right)^{2}+\text { higher derivatives }\right], \\
\bar{f}_{\mu \nu}=\bar{\partial}_{\mu} \bar{a}_{\nu}-\bar{\partial}_{\nu} \bar{a}_{\mu}, \bar{\partial}^{2}=\partial_{0}^{2}+\bar{\partial}_{1}^{2}, \bar{a}_{\mu}=\bar{\partial}_{\mu} \lambda(\bar{x})+\epsilon_{\mu \nu} \bar{\partial}_{\nu} \phi(\bar{x}), r^{2}=v_{0}^{2} / v_{1}^{2},
\end{gathered}
$$

and the inverse of the Dirac operator is written as,

$$
\begin{aligned}
& \left\langle x\left|\left[\gamma_{\mu}\left(\bar{p}^{\mu}+\bar{a}^{\mu}\right)\right]^{-1}\right| y\right\rangle=e^{i \lambda(\bar{x})-\gamma_{5} \phi(\bar{x})} S_{0}(x-y) e^{-i \lambda(\bar{y})-\gamma_{5} \phi(\bar{y})}, \\
& S_{0}(x-y)=\left\langle x\left|\left[\gamma_{\mu} \bar{p}^{\mu}\right]^{-1}\right| y\right\rangle, \gamma_{5}=-i \gamma_{0} \gamma_{1}=\left(\begin{array}{cc}
-1 & 0 \\
0 & 1
\end{array}\right) .
\end{aligned}
$$


Combining Eq.(21) and Eq.(22), we have

$$
\begin{gathered}
\left\langle\psi(x) \psi^{\dagger}(y)\right\rangle_{x_{0}=y_{0}}=\frac{1}{N} \int d \lambda d \phi e^{-S_{e f f}(\lambda, \phi)} e^{i(\lambda(\bar{x})-\lambda(\bar{y}))-\gamma_{5}(\phi(\bar{x})-\phi(\bar{y}))} \times \\
\left\{\cos p_{F}(y-x) \operatorname{Tr}\left[\gamma_{0} S_{0}(x-y)\right]+\sin p_{F}(y-x) \operatorname{Tr}\left[\gamma_{1} S_{0}(x-y)\right]\right\} \\
=\frac{\sin p_{F}|y-x|}{\pi(x-y)^{\beta_{F}}}, \beta_{F}=1-\frac{N}{4 \pi}\left[\frac{\xi-1}{\xi(\xi+1)}\left(\frac{C}{v_{1}^{2}}-\frac{C}{v_{0}^{2}}\right)-\frac{1}{2 \pi \xi}\right] \\
\langle\rho(x) \rho(y)\rangle_{x_{0}=y_{0}}=\text { const }+\operatorname{Tr}\left(\gamma_{0} S_{0}(x-y) \gamma_{0} S_{0}(y-x)\right) \\
+\frac{1}{N} \int d \lambda d \phi e^{-S_{e f f}(\lambda, \phi)-2 \gamma_{5}(\phi(x)-\phi(y))} S_{0}(x-y) S_{0}(y-x) \cos 2 p_{F}(x-y) \\
=\text { const }+\frac{1}{2 \pi^{2}(x-y)^{2}}-\frac{\cos 2 p_{F}(x-y)}{2 \pi^{2}(x-y)^{\alpha}}, \\
\alpha=2-\frac{N}{\pi}\left[\frac{C}{\xi v_{1}^{2}}+\frac{1}{\xi(\xi+1)}\left(\frac{C}{v_{0}^{2}}-\frac{C}{v_{1}^{2}}\right)\right]
\end{gathered}
$$

where

$$
N=\frac{v_{1}^{2}}{C} \frac{1}{C / v_{0}^{2}+1 / 2 \pi}, \xi=\sqrt{\frac{v_{1}^{2}+2 \pi C}{v_{0}^{2}+2 \pi C}} .
$$

Exponents $\alpha$ and $\beta_{F}$ satisfy Luttinger liquid relations [5 but their $g$ dependence are different from those obtained by Kawakami and Yang [3] based on conformal field theory. The reason for this $g$ dependence is due to the coupling dependent kinetic term, $F\left(p_{1}\right)$, in Eq.(10) and (20). This term does not exist and $\beta_{F}$ goes to infinity as $g$ goes to infinity in short range potential models.

(B) Self-consistent stationary phase approximation - In the previous part, we see that the mean field $U_{0}\left(x_{1}, y_{1}\right)$ of Eq.(8) is a solution of classical equation of motion, Eq.(7). However, it does not make the total free energy stationary due to the higher order correction as is seen in Eq.(23). Especially the long distance behavior of propagator is very different from the free propagator. In this section we find a self-consistent mean field that includes the long distance fluctuations, and we compute current correlation functions under the self-consistent mean field. The mean field now satisfies

$$
\begin{gathered}
\int \mathcal{D} U\left\{U-\left\langle\psi^{\dagger} \psi\right\rangle_{U}\right\} e^{-S_{e f f}(U)}=0, \\
\langle U\rangle=\tilde{U}_{0} .
\end{gathered}
$$

We start from a modified bi-local mean field $\tilde{U}_{0}$ which incorpolates the radiative correction in the long distance region and compute the correction to the propagator. With a suitable $\tilde{U}_{0}$, the full propagator agrees to the initial $\tilde{U}_{0}$ and full self-consistency condition is satisfied. We find such $\tilde{U}_{0}$ first and compute other quantities next. 
We start from the following form of the $\tilde{U}_{0}$ :

$$
\tilde{U}_{0}(r)= \begin{cases}\frac{\sin p_{F} r}{\pi r}\left(\frac{r}{r_{0}}\right)^{1-\beta_{F}}, & r_{0}<|r| \\ \frac{\sin p_{F} r}{\pi r}, & |r| \leq r_{0}\end{cases}
$$

which has a fractional power in the long distance region and normalized to the density $\rho$ at the origin, and substitute it to Eq.(16). A parameter $\beta_{F}$ is unknown in the beginning and is determined from the full self-consistency condition. Another parameter $r_{0}$ is connected with a short distance dynamics but is regarded as a constant in this paper.

We repeat the same procedure as before. Namely we first expand the bi-local field with a new complete set of functions $\left\{\tilde{q}_{n}(r)\right\}$ which satisfies,

$$
\int d r \frac{V(r)}{4}\left[\left|\tilde{U}_{0}(r)\right|^{2} \tilde{q}_{n}(r) \tilde{q}_{m}(-r)-\tilde{U}_{0}(0)^{2} \tilde{q}_{n}(0) \tilde{q}_{m}(0)\right]=\delta_{n, m}
$$

as

$$
U\left(x_{1}, y_{1} ; x_{0}\right)=\tilde{U}_{0}\left(x_{1}-y_{1}\right)\left[1+\sum_{n=0}^{\infty} \tilde{a}_{n}\left(\frac{x_{1}+y_{1}}{2} ; x_{0}\right) \tilde{q}_{n}\left(x_{1}-y_{1}\right)\right] .
$$

The action is expressed with local fields $\tilde{a}_{n}$ in the same manner as Eq.(15) with couplings $F_{l}(p)$ obtained by replacing $U_{0}$ with $\tilde{U}_{0} . F_{l}\left(p_{F}\right)$ behaves as,

$$
F_{l}\left(p_{F}\right)=\text { const } \epsilon^{\frac{1}{4}}, \quad\left(l \geq \beta_{F}+\frac{1}{2}\right) .
$$

Small $\epsilon$ limit of $F_{l}\left(p_{F}\right)$ depends on the exponent $\beta_{F} . F_{l}\left(p_{F}\right)$ vanishes in the small $\epsilon$ limit if $l \geq \beta_{F}+\frac{1}{2}$. Hence the number of local fields that couple with the fermionic system in this limit depends on the $\beta_{F}$. It is necessary to treat the effective Lagrangian separately depending on the magnitude of $\beta_{F}$.

(i) $1 \leq \beta_{F}<\frac{3}{2}$ : For this value of $\beta_{F}$ two local fields $\tilde{a}_{0}(x)$ and $\tilde{a}_{1}(x)$ couple with fermion and the others decouple in the $\epsilon \rightarrow 0$ limit. Hence the situation is the same as $\beta_{F}=1$ case.

(ii) $\frac{3}{2} \leq \beta_{F}<2$ : In this case three local fields $\tilde{a}_{0}(x), \tilde{a}_{1}(x)$, and $\tilde{a}_{2}(x)$ couple. The interaction parts are given by,

$$
\begin{gathered}
\sum_{n=0}^{2} \int d x \psi^{\dagger}(x) F_{n}(p) \psi(x) \tilde{a}_{n}(x), \\
F_{n}(p)=2 \int d r\left\{\tilde{U}_{0}(r) \tilde{q}_{n}(r) e^{i p r}-\tilde{U}_{0}(0) \tilde{q}_{n}(0)\right\} \frac{V(r)}{4} .
\end{gathered}
$$

The couplings $F_{n}(p)$ are approximated with the Fermi momentum value $F_{n}\left( \pm p_{F}\right)$ as far as the long wave length physics is concerned. The couplings $F_{n}\left( \pm p_{F}\right)$ are even functions if $n$ is even and are odd functions if $n$ is odd,

$$
F_{2}\left(-p_{F}\right)=F_{2}\left(p_{F}\right)
$$




$$
F_{1}\left(-p_{F}\right)=-F_{1}\left(p_{F}\right) .
$$

We find two component field representation of the interaction part as,

$$
\begin{gathered}
\bar{\psi}(x) \gamma_{0} \tilde{F}_{0}\left(p_{F}\right) \psi(x) b_{0}(x)+\bar{\psi}(x) \gamma_{1} F_{1}\left(p_{F}\right) \psi(x) b_{1}(x), \\
\tilde{F}_{0}\left(p_{F}\right)=\sqrt{F_{0}\left(p_{F}\right)^{2}+F_{2}\left(p_{F}\right)^{2}}, \\
b_{0}(x)=\frac{F_{0}\left(p_{F}\right)}{\tilde{F}_{0}\left(p_{F}\right)} \tilde{a}_{0}(x)+\frac{F_{2}\left(p_{F}\right)}{\tilde{F}_{0}\left(p_{F}\right)} \tilde{a}_{2}(x) .
\end{gathered}
$$

With the other component $b_{1}(x)$ and $b_{2}(x)$ defined as

$$
b_{1}(x)=\tilde{a}_{1}(x), \quad b_{2}(x)=-\frac{F_{2}\left(p_{F}\right)}{\tilde{F}_{0}\left(p_{F}\right)} \tilde{a}_{0}(x)+\frac{F_{0}\left(p_{F}\right)}{\tilde{F}_{0}\left(p_{F}\right)} \tilde{a}_{2}(x),
$$

the mass term has the equivalent form as before, $b_{0}^{2}+b_{1}^{2}+b_{2}^{2}=\tilde{a}_{0}^{2}+\tilde{a}_{1}^{2}+\tilde{a}_{2}^{2}$.

(iii) $2 \leq \beta_{F}$ : More fields couple with fermionic system. In the long distance region interaction terms are written into the two component form as,

$$
\bar{\psi}(x) \gamma_{0} \tilde{F}_{0}\left(p_{F}\right) \psi(x) b_{0}(x)+\bar{\psi}(x) \gamma_{1} \tilde{F}_{1}\left(p_{F}\right) \psi(x) b_{1}(x),
$$

where,

$$
\begin{gathered}
\tilde{F}_{0}\left(p_{F}\right)=\sqrt{\sum_{n=e v e n} F_{n}^{2}}, \quad \tilde{F}_{1}\left(p_{F}\right)=\sqrt{\sum_{n=o d d} F_{n}^{2}}, \\
b_{0}(x)=\frac{1}{\tilde{F}_{0}\left(p_{F}\right)}\left\{\sum_{n} \tilde{F}_{2 n}\left(p_{F}\right) \tilde{a}_{2 n}(x)\right\}, \\
b_{1}(x)=\frac{1}{\tilde{F}_{0}\left(p_{F}\right)}\left\{\sum_{n} \tilde{F}_{2 n+1}\left(p_{F}\right) \tilde{a}_{2 n+1}(x)\right\} .
\end{gathered}
$$

Thus the fields $b_{0}(x)$ and $b_{1}(x)$ couple with the fermion in the long distance region with the effective coupling strength $\tilde{F}_{0}\left(p_{F}\right)$ and $\tilde{F}_{1}\left(p_{F}\right)$ and the other fields decouple. Consequently, the effective Lagrangian has the equivalent form to that of the previous case.

Now we solve the full self-consistency condition with the effect of the low energy and long wave fluctuations around the mean field included. We have the self-consistency conditions, from Eq.(25),

$$
\begin{aligned}
\left\langle b_{n}\right\rangle & =0, \\
\beta_{F}(\text { output }) & =\beta_{F}(\text { input }),
\end{aligned}
$$

We find the exponent $\beta_{F}$ numerically as a function of the coupling constant $g$. The exponent $\nu=\beta_{F}-1$ thus obtained for $p_{F} r_{0}=1,0.1$ is shown in Fig.1.

At the end we compute the ground state energy that includes corrections from the low energy and long wave length fluctuations. Since the partition function at the low 
temperature is expressed as $\exp \left(-\beta E_{0}\right)$ with the ground energy $E_{0}$, the ground state energy is found from the partition function. We find the energy in the lowest order from the $\mathcal{Z}_{0}$ and its correction from the $\tilde{\mathcal{Z}}$. We use the linerized form of the Lagrangian. This Lagrangian is good in $p_{1} \leq p_{F}$. The energy density is expressed as

$$
\begin{gathered}
\mathcal{E}_{0}=\frac{p_{F}^{3}}{6 \pi m}-\int d r \frac{V(r)}{4}\left\{\tilde{U}_{0}(r)\left(2 U_{0}(r)-\tilde{U}_{0}(r)\right)-\frac{p_{F}^{2}}{\pi^{2}}\right\}, \\
\Delta \mathcal{E}=\frac{1}{8 \pi^{2}} \int d p_{0} d p_{1} \log \left[1+\frac{F_{0}^{2} p_{0}^{2}+F_{1}^{2} p_{1}^{2} C^{2}-\tilde{q}_{0}^{2} F_{1}^{2} C^{2} p_{1}^{2} C_{0}\left(p_{1}\right)}{4 \pi C\left(p_{0}^{2}+\left(C p_{1}\right)^{2}\right)\left(1-\tilde{q}_{0}^{2} C_{0}\left(p_{1}\right)\right)}\right] .
\end{gathered}
$$

The expression of $\Delta \mathcal{E}$ is valid in the low energy region. We specify the region of integration as $\left|p_{1}\right| \leq p_{F},\left|p_{0}\right| \leq C p_{F}$. The mean field energy density $\mathcal{E}_{0}$ is given in Fig.2, and the energy density $\mathcal{E}_{0}+\Delta \mathcal{E}$ from these regions are given in Fig.3. In the same figure, the exact energy value obtained by Sutherland is given. We see that our value is close to the exact value. Thus long wave fluctuations are responsible to the most part of the ground state energy.

As a conclusion, we see that the one dimensional system with long range $1 / r^{2}$ interaction can be expressed with the self-consistent bi-local mean field theory. The ground state energy agrees well with, but the exponents of the correlation functions deviate from the exact value if the linearized form is used.

The present work is partially supported by a Grant-in-Aid for general Scientific Research (03640256), and the Grant-in-Aid for Scientific Research on Priority Area (04231101), the Ministry of Education, Science and Culture, Japan.

\section{References}

[1] B.Sutherland, J.Math.Phys.12(1971)246,251;

Phys.Rev.A4(1971)2019,ibid,A5(1971)1372.

[2] F.Calogero, J.Math.Phys.10(1969)2191.

[3] N.Kawakami and S.-K.Yang, Phys.Rev.Lett.67(1991)2493,

See a review in Prog.Theor.Phys. Suppl.107(1992)59.

[4] J.M.Luttinger, J.Math.Phys.4(1963)1154;

S.Tomonaga, Prog.Theor.Phys.5(1950)544.

[5] F.D.M.Haldane, Phys.Rev.Lett.45(1980)1358, ibid,47(1981)1840.

[6] D.C.Mattis and E.H.Lieb, J.Math.Phys.6(1965)304;

A.Luther and I.Peschel, Phys.Rev.B9(1974)2911. 
[7] R.B.Laughlin, Phys.Rev.Lett.50(1983)1395

[8] K.Ishikawa, Prog.Theor.Phys.Suppl.107(1992)167; Prog.Theor.Phys.88(1992)881; K.Ishikawa and N.Maeda, "A Mean Field Theory for the Quantum Hall Liquid II, -The Vortex Solution-", Hokkaido University preprint EPHOU 93-001.

[9] R.L.Stratonovich, Soviet Phys. Doklady,2(1957)416;

J.Hubbard, Phys.Rev.Lett.3(1959)77.

\section{Figure Captions}

Fig.1 : The exponent $\nu=\beta_{F}-1$ is given as a function of the coupling constant. The dash line shows the value obtained from conformal field theory and dotts show the selfconsistent mean field values for $p_{F} r_{0}=0.1$ and $p_{F} r_{0}=1.0$. The real line shows the simple mean field value.

Fig.2 : The ground state energy density is given. The real line shows the simple mean field value, and the dotts show the self-consistent mean field values for $p_{F} r_{0}=0.1$ and $p_{F} r_{0}=1.0$. The dash line is the exactly known value.

Fig.3 : The ground state energy density with fluctuations included are given. The real line shows the simple mean field value, and the dotts show the self-consistent mean field values for $p_{F} r_{0}=0.1$ and $p_{F} r_{0}=1.0$. The dash line is the exactly known value. 